\section{SCIENCE CHINA \\ Physics, Mechanics \& Astronomy}

\title{
Few-layer metasurfaces with engineered structural symmetry
}

\author{
ZhanCheng $\mathrm{Li}^{1}$, Hua Cheng ${ }^{1}$, and ShuQi Chen ${ }^{1,2,3^{*}}$ \\ ${ }^{1}$ Key Laboratory of Weak Light Nonlinear Photonics, Ministry of Education, School of Physics and TEDA Institute of Applied Physics, \\ Nankai University, Tianjin 300071, China; \\ ${ }^{2}$ The Collaborative Innovation Center of Extreme Optics, Shanxi University, Taiyuan 030006, China; \\ ${ }^{3}$ Collaborative Innovation Center of Light Manipulations and Applications, Shandong Normal University, Jinan 250358, China
}

Received February 3, 2021; accepted March 8, 2021; published online April 2, 2021

Citation: Z. C. Li, H. Cheng, and S. Q. Chen, Few-layer metasurfaces with engineered structural symmetry, Sci. China-Phys. Mech. Astron. 64, 264231 (2021), https://doi.org/10.1007/s11433-021-1687-x

\begin{abstract}
Metasurfaces are planar arrays composed of artificial nanostructures at the subwavelength scale. Since the first demonstration of their excellent capacity in the phase manipulation of optical waves, metasurfaces have attracted increasing attention in optics and other disciplines, with extraordinary progress over the past decade. The unique capabilities of metasurfaces in the multidimensional manipulation of optical waves have made them versatile and powerful platforms for integrating and minimizing optical devices $[1,2]$. However, as planar arrays, the insufficient design freedoms of the structural symmetry of metasurfaces have led to certain defects in their controllability and functionality, impeding their further development in integrated photonics, quantum optics, and other fields. Recently, fewlayer metasurfaces comprising more than one functional layer have become promising alternatives to overcome the drawbacks in metasurfaces [3]. Few-layer metasurfaces cannot be treated as simple stacks of multiple metasurfaces. The abundant interlayer light-matter interactions within fewlayer metasurfaces and the high design freedoms of fewlayer metasurfaces have expanded the design flexibility significantly, resulting in optical devices with high efficiency and novel optical functionalities. Notably, compared with planar metasurfaces which always have a mirror symmetry with respect to the plane, researchers can arbitrarily design
\end{abstract}

*Corresponding author (email: schen@nankai.edu.cn) the structural symmetry of few-layer metasurfaces. This key feature enables few-layer metasurfaces to achieve some optical responses that planar metasurfaces cannot.

Implementation of giant three-dimensional (3D) chiral optical responses in few-layer metasurfaces is a major research subject in nanophotonics [4]. Compared with the twodimensional chiral optical responses in planar metasurfaces, which are attributed to the circular polarization conversion effect, the chiral optical responses of few-layer metasurfaces with unique structural symmetry designs can be purely 3D. This suggests that few-layer metasurfaces can exhibit orthogonal circular polarization eigenstates. For example, Li et al. [5] proposed a bilayer metasurface with $C_{4 v}$ structural symmetry but without additional reflection symmetry to realize the spin-selective transmission of optical waves. Further research proved that rotational disorder can be used to realize a pure $3 \mathrm{D}$ chiral optical response in few-layer metasurfaces [6]. Few-layer metasurfaces with giant 3D chiral optical responses are a versatile platform for examining chirality with potential applications in chiral sensing.

Asymmetric transmission of linear-polarized waves, which cannot be realized in planar metasurfaces, can be easily implemented in few-layer metasurfaces because the structural symmetry of few-layer metasurfaces can be arbitrarily designed [7]. Recently, benefiting from the asymmetric 
transmission effects of optical waves in few-layer metasurfaces and their high effciency, the few-layer metasurfaces with unique structural symmetry designs have become a good approach for bidirectioanl independent manipulation of optical waves, which are widely used for asymmetric holograms, asymmetric wavefront manipulation, and wavevector selected multifunctional integration [8,9]. Recent advances in few-layer metasurfaces have demonstrated that few-layer metasurfaces with unique structural symmetry designs can be further used to realize the chiral bound states in the continuum, full-space manipulation of optical waves, and polarization-selected multifunctional integration [10]. These approaches effectively show the unprecedented possibilities of few-layer metasurafaces for the bidirectional and multidimensional manipulation of optical waves.

In conclusion, the few-layer metasurfaces with engineered structural symmetry have a superior ability to manipulate optical waves. Therefore, few-layer metasurfaces with high design freedoms will play an increasingly important role in nanophotonics.

1 S. Chen, Z. Li, W. Liu, H. Cheng, and J. Tian, Adv. Mater. 31, 1802458 (2019).

2 T. Li, Sci. China-Phys. Mech. Astron. 63, 284231 (2020).

3 Z. Li, W. Liu, H. Cheng, and S. Chen, Sci. China-Phys. Mech. Astron. 63, 284202 (2020).

4 W. Du, X. Wen, D. Gérard, C. W. Qiu, and Q. Xiong, Sci. China-Phys. Mech. Astron. 63, 244201 (2020).

5 Z. Li, W. Liu, H. Cheng, S. Chen, and J. Tian, Sci. Rep. 7, 8204 (2017).

6 S. Fasold, S. Lin $\beta$, T. Kawde, M. Falkner, M. Decker, T. Pertsch, and I. Staude, ACS Photon. 5, 1773 (2018).

7 C. Menzel, C. Helgert, C. Rockstuhl, E. B. Kley, A. Tünnermann, T. Pertsch, and F. Lederer, Phys. Rev. Lett. 104, 253902 (2010), arXiv: 1005.1970.

8 K. Chen, G. Ding, G. Hu, Z. Jin, J. Zhao, Y. Feng, T. Jiang, A. Alù, and C. W. Qiu, Adv. Mater. 32, 1906352 (2020).

9 D. Frese, Q. Wei, Y. Wang, L. Huang, and T. Zentgraf, Nano Lett. 19, 3976 (2019).

10 Y. Yuan, K. Zhang, B. Ratni, Q. Song, X. Ding, Q. Wu, S. N. Burokur, and P. Genevet, Nat. Commun. 11, 4186 (2020). 